Revista Interdisciplinaria de Humanidades, Educación, Ciencia y Tecnología

Año VII. Vol. VII. N³3. Edición Especial III. 2021

Hecho el depósito de ley: pp201602FA4721

ISSN-L: 2542-3029; ISSN: 2610-802X

Universidad Nacional Experimental Francisco de Miranda (UNEFM). Santa Ana de Coro. Venezuela

Cristian Fernando Álvarez-Guamán; Juan Carlos Erazo-Álvarez

$\underline{\text { DOI } 10.35381 / \mathrm{cm} . v 7 i 3.578}$

\title{
Gamificación en el proceso de enseñanza de algebra: una experiencia con Educaplay
}

Gamification in the algebra teaching process: an experience with Educaplay

\author{
Cristian Fernando Álvarez-Guamán \\ cfalvarezg30@est.ucacue.edu.ec \\ Universidad Católica de Cuenca, Azogues \\ Ecuador \\ https://orcid.org/0000-0001-7604-6572 \\ Juan Carlos Erazo-Álvarez \\ jcerazo@ucacue.edu.ec \\ Universidad Católica de Cuenca, Cuenca \\ Ecuador \\ https://orcid.org/0000-0001-6480-2270
}

Recibido: 15 de agosto de 2021

Aprobado: 15 de noviembre de 2021 


\title{
RESUMEN
}

La investigación tiene por objeto analizar de qué manera la Herramienta Educaplay puede aportar en el proceso de aprendizaje de algebra en los estudiantes de Octavo Año de Básica de la Unidad Educativa "Mariscal Antonio José de Sucre"; por lo tanto, se realiza una investigación de tipo explicativa. En consecuencia, mediante un análisis de los datos en el programa SPSS con la prueba t student se llega a la conclusión que la incorporación de Educaplay en el aprendizaje de algebra mejoro el nivel de asimilación de conocimientos por parte de los alumnos. La propuesta desarrollada frente a esta situación es el diseño de una guía metodológica para los docentes, con el propósito de que implementen la utilización de la plataforma en las aulas de clase.

Descriptores: Enseñanza multimedia; enseñanza audiovisual; técnica didáctica. (Palabras tomadas del Tesauro UNESCO).

\begin{abstract}
The research aims to analyze how the Educaplay Tool can contribute to the algebra learning process in Eighth Year Elementary students of the "Mariscal Antonio José de Sucre" Educational Unit; therefore, a explicative investigation is carried out. Consequently, through an analysis of the data in the SPSS program with the student $t$ test, it is concluded that the incorporation of Educaplay in the learning of algebra improved the level of assimilation of knowledge by the students. The proposal developed in the face of this situation is the design of a methodological guide for teachers, with the purpose that they implement the use of the platform in classrooms.
\end{abstract}

Descriptors: Multimedia instruction; audiovisual instruction; classroom techniques. (Words taken from the UNESCO Thesaurus). 


\section{INTRODUCCIÓN}

Los países que han efectuado un uso correcto de las tecnologías de la información, obtuvieron una mejora en sus sistemas educativos, tanto en el aprovechamiento académico, como la trasmisión de experiencias y conocimientos. Según un estudio realizado por la Organización de las Naciones Unidas Para la Educación la Ciencia y la Cultura [UNESCO] (2015) sobre la utilización de herramientas móviles para el proceso de enseñanza a alumnos del octavo al undécimo año de zonas rurales en Miramar; obtuvo como resultado que existe un cambio importante en los educandos al mejorar su interés por aprender. Además, esta herramienta digital desarrolla confianza entre los docentes por la didáctica con la que se ejecuta para el proceso de enseñanza.

En este sentido, este trabajo evidencia que la utilización de recursos tecnológicos educativos posibilita actualizar los procesos de enseñanza; es decir, dejar a un lado la utilización de papel y lápiz, para optar por la utilización de herramientas que generan interés de los educandos a través del juego que otorga la Gamificación. De la misma forma, debido a la pandemia, la Organización para la Cooperación y el Desarrollo Económico [OCDE] (2021) recalca que es indispensable el uso de plataformas digitales con los alumnos; ya que estas se vuelven indispensables en estos momentos del planeta debido al cierre de las escuelas; consideran que su utilización engloba una variedad de oportunidades, en consecuencia, dinamizan los entornos de aprendizaje.

Por lo tanto, es prudente considerar que el desarrollo de este trabajo es importante; puesto que, el uso de plataformas como Educaplay permite demostrar que utilizar la Gamificación como metodología de enseñanza, conduce a la asimilación de conocimientos y posibilita lograr una mejor comprensión e interés por parte de los educandos. Según los autores Holguín-García et al. (2020) la Gamificación incide en el aprovechamiento académico siempre que este diseñada en parámetros cognitivos adecuados.

Por otra parte, en lo que respecta a nivel nacional, la problemática se da porque tanto el gobierno como el Ministerio de Educación, han sido indiferentes respecto a la inclusión 
de este tipo de herramientas educativas en las instituciones nacionales. Solo en los últimos años a raíz de la pandemia del Covid-19, se puede observar un pequeño intento de implementar recursos educativos tales como Zoom, Teams, Moodle, etc. Sin embargo, esto no quiere decir que existe un dominio de los docentes de este tipo de herramientas, además de una mejora en el desempeño académico por parte de los educandos.

Puesto que; a nivel nacional se puede observar mediante los estudios realizados en la aplicación de las pruebas del Programa Internacional para la Evaluación de Instituciones [PISA-D] (2018) que según los informes de los resultados; existe un bajo rendimiento de los alumnos en las asignaturas primordiales como Matemática, Ciencias Naturales, Lengua y Literatura y Estudios Sociales. En lo que respecta a la asignatura de Matemática en la institución sujeta a estudio, todo esto nace como consecuencia de diferentes factores tales como; el desconocimiento del lenguaje algebraico, fallas en operaciones básicas, metodologías de enseñanza tradicionales que influyen en el bajo desempeño y en la falta de interés de los alumnos.

Por lo antes expuesto, el propósito de este documento investigativo es analizar como la Herramienta Educaplay, puede aportar en el proceso de aprendizaje de algebra en los estudiantes de Octavo Año de Básica, pertenecientes a la Institución Educativa "Mariscal Antonio José de Sucre" de la parroquia Rivera en la comunidad de Zhoray. Por ende; para lograrlo se desarrolla una aplicación de campo y una revisión minuciosa de literatura científica que fundamenta con datos y resultados los beneficios de incorporar este tipo de herramienta en la trasmisión de conocimientos de modo que generen un aprendizaje innovador e integral.

\section{Referencial teórico}

En el siguiente apartado se describe los aportes científicos en función de las variables de investigación, tomando como fuentes de información artículos científicos provenientes de base de datos como Redalyc y Scielo. De la misma manera; se toma como datos a documentos expuestos por organismos nacionales e internacionales en el periodo de 
tiempo 2015-2021, con el propósito de tener un panorama de la situación a nivel internacional, regional y nacional. Para la estructuración de este documento se utiliza el denominado formado IMRYD, en el cual Lam-Díaz (2016) plantea tomar las iniciales de los enunciados fundamentales de un artículo; Es decir, introducción, metodología, resultados y discusión.

En primera instancia a nivel internacional, los autores Vaillant et al. (2020) en Uruguay realizaron una investigación que buscaba analizar la utilización de plataformas educativas en la enseñanza de matemática en secundaria. Explicaron que se desarrolló la aplicación de una encuesta online de tipo Likert, obteniendo como resultado que las herramientas más utilizadas son GeoGebra con un porcentaje del 55,7\% y la Plataforma Adaptativa de Matemática PAM con un 49,8\%. Además, los medios digitales más utilizados son los teléfonos inteligentes seguido de las computadoras. Finalmente, los autores indican que la variedad de recursos que ofrece una plataforma permite desarrollar diferentes habilidades tecnológicas.

De igual modo, en lo referente a un estudio internacional Labrador Ruiz de la Hermosa y Villegas-Portero (2016) en España, mediante su trabajo de investigación buscaron desarrollar una metodología que mejore tanto la parte critica como la creativa del alumno por medio de plataformas digitales gamificadoras que diseñan perfiles informáticos. Demostraron mediante la aplicación de encuestas que la incorporación de la Gamificación, da como consecuencia que los usuarios obtengan mejores resultados con una tasa de aceptación prometedora; puesto que, en lo que respecta a las notas parciales se observó un incremento considerable, al igual que en las notas anuales.

También, Ortiz-Colón et al. (Ortiz-Colón et al., 2018) en España, explican que la Gamificación en plataformas orientadas a la educación sirve para dar respuesta a las necesidades de las nuevas generaciones, debido a que buscan motivación dentro del contexto educativo para su aprendizaje autónomo. El trabajo que realizaron mediante una metodología cuantitativa está basado en el análisis de contenidos de los beneficios que otorga la Gamificación; por ende, se tomaron como muestra 37 experiencias cuyo eje 
principal fue el beneficio de la incorporación de la competencia en un juego gamificada, teniendo como resultado que el $75 \%$ del alumnado obtuvo motivación por aprender mediante esta metodología.

Por su parte, en lo que respecta a nivel Regional, Boude-Figueredo y Sarmiento (2016) en Colombia para su trabajo de investigación que toma como base la utilización de la web 2.0 en la enseñanza de alumnos colombianos. Encontraron como resultados que la aplicación de la herramienta Educaplay como recurso tecnológico para el aprendizaje, influye tanto en los alumnos como en los docentes; puesto que, al realizar un análisis cuantitativo-descriptivo de los efectos de plataforma en la educación de este país; se encontró que en lo que respecta al nivel de educación media, tanto las plataformas como la web 2.0 afianzan los aprendizajes del aula, mientras que a los docentes les facilita la aplicación de proyectos interdisciplinarios.

Asimismo, en Perú Cencia-Crispín et al. (2021) elaboraron una investigación sobre el uso de herramientas de libre acceso como Educaplay, Google Drive, Suite y Genially con el objetivo de determinar las percepciones de los profesores universitarios para su uso en actividades lúdicas de docencia en tiempos de Covid-19; encontrando como resultado que las herramientas proporcionan una mayor facilidad como respuesta de apoyo hacia el docente. De esta manera; mediante el estudio se demostró que los profesionales en la docencia deben estar preparados para la cambiante era digital; puesto que, debido a situaciones como la pandemia, tanto la trasmisión de conocimiento, como la metodología pedagógica obligatoriamente deben incorporar herramientas innovadoras.

De igual modo, Ducuara-Amado et al. (2020) en Colombia, mediante el trabajo de investigación sobre material gamificado, para la enseñanza de ecología a través de sus conceptos fundamentales en educación media; propusieron realizar un juego en Educaplay para un estudio cuantitativo de recolección de datos numéricos, con el fin de saber la aceptación que tuvo el material en los estudiantes de décimo grado del instituto técnico de Santo Tomas en Boyacá; se tomó una muestra de 30 alumnos, teniendo una 
aceptación del 95\% en la aplicación de juegos como estrategia de Gamificación para materiales didácticos.

Mientras tanto; (Salinas-Gañango \& Salvati, 2020), utilizan como recurso didáctico Educaplay en la enseñanza de física y química explican que; al utilizar el método inductivo-deductivo en la aplicación pruebas objetivas mediante un pre test y pos test ; esta herramienta permite al docente incrementar el aprendizaje del alumnado; puesto que, de los 22 estudiantes del segundo semestre de la carrera de biología que sirvieron como muestra, en su mayoría los resultados obtenidos fueron mejores que al aplicar metodologías tradicionales.

También, Collaguazo-Alvarez y Maggi-Barba (2020) en Ecuador, propusieron un artículo sobre la utilización del recurso informático Educaplay para aprender sobre moléculas mediante un enfoque cualitativo en dos grupos de estudiantes, con el objetivo de realizar una evaluación formativa de las actividades realizadas en Educaplay; comprobando que los resultados mediante la prueba $Z$, indicaban que los alumnos que utilizaron esta plataforma, superan en rendimiento a los alumnos que no la utilizaron. Con la muestra de 32 estudiantes; el 46,88\% alcanzan los aprendizajes requeridos, mientras que el 50\% están próximos a alcanzar; por último, solo el 3,12\% no alcanzan los aprendizajes.

Por otra parte, Holguín-García et al. (2020) en Ecuador, mediante su investigación direccionada a la Gamificación para la enseñanza de matemática , cuyo objetivo era evaluar la influencia de aplicaciones gamificadoras en el rendimiento de la asignatura; obtuvieron como resultados que siempre que el software y la técnica tengan parámetros cognitivos, otorgaran mejoras significativas en cuanto al nivel educativo, competencias, ventajas y aprovechamiento académico de los estudiantes; puesto que, los mantiene estimulados durante todo el proceso de aplicación, lo que les posibilita reforzar conocimientos de forma dinámica.

Finalmente, después de revisar varios autores con sus diferentes estudios, tanto de la Gamificación como de la utilización de plataforma Educaplay en diferentes áreas. Además de su utilización desde diferentes perspectivas metodológicas; la aplicación de 
esta herramienta es oportuna en el momento en que atraviesa nuestra sociedad al estar inmersos en la virtualidad, Además, cuenta con un sinnúmero de alternativas para su aplicación en varios subniveles de educación; De igual modo, al paso que avanza la tecnología, los docentes deben estar preparados e innovar para facilitar el proceso de enseñanza aprendizaje.

\section{Referencial teórico}

Las exigencias en la sociedad actual debido a la pandemia y al avance acelerado de las Tecnologías de la Información y Comunicación, obligan a que el docente tenga que buscar metodologías innovadoras en el aula. En este sentido; el proceso de incorporar plataformas digitales para el aprendizaje de Matemática, posibilita a que el alumnado muestre un interés en las actividades educativas, más aún, en asignaturas de difícil comprensión como algebra, por ende, para el desarrollo de este epígrafe se considera primordial definir las siguientes líneas de investigación.

En primer lugar, es importante puntualizar la definición de algebra, según Baldor (2010) "es la rama de la Matemática que estudia la cantidad considerada del modo más general posible" (p. 5). Por lo tanto, el álgebra es una parte de la Matemática que utiliza simbologías y expresiones algebraicas tales como; variables, exponentes; coeficientes, signos; con el propósito de relacionarlas mediante operaciones aritméticas que posibilitan el desarrollo de ecuaciones, que permiten entender mediante formulaciones lógicas el funcionamiento del universo.

Asimismo, Faria y Maltempi (2019) explican que la Matemática aplicada en forma didáctica permite trabajar de manera eficaz mediante la utilización de software dinámico que relaciona los saberes con la enseñanza, cumpliendo con el objetivo de desarrollar el pensamiento lógico del educando mediante la resolución de problemas que se pueden manifestar dentro o fuera de un entorno. De esta manera, mediante herramientas como GeoGebra se logra profundizar áreas como geometría, algebra y aritmética; conocidas por presentar una mayor dificultad para su comprensión. Por último, los autores 
concluyen que los educadores encontraran varias ventajas al aplicar sus clases con este tipo de herramienta.

Mientras tanto, en lo que tiene que ver con recursos tecnológicos, para los autores BadiaGarganté et al. (2015) son aquellos medios digitales que se basan en la tecnología de la red, para cumplir con el objetivo de facilitar las actividades del diario vivir de la sociedad, así como de diferentes tipos de instituciones ya sean estas públicas o privadas. En lo que respecta al sector educativo, estos recursos ayudan a la utilización de metodologías didácticas diseñadas para la interacción entre docentes y alumnos en de forma online; en consecuencia, facilitan la comprensión de determinado tema o refuerzan la comprensión del mismo.

Por otro lado, en lo que respecta a metodologías didácticas que estén orientadas a la educación, las mismas apoyan de manera significativa en el proceso de enseñanza y en la trasmisión de conocimientos que son necesarios en la práctica docente. Según VargasMurillo (2017) permiten mejorar el proceso de enseñanza del docente; puesto que, optimizan a través de la Gamificación el aspecto pedagógico en los educandos, de esta manera generan interés al provocar una comunicación asertiva, permitiendo un aprendizaje autónomo e integral. Entre los tipos de recursos didácticos tenemos:

- Material Audiovisuales

- Textos Impresos

- Pizarras: tradicional, didáctica

- Tecnologías de la información; Software, Internet

- Plataformas educativas

También, es necesario definir lo que son las plataformas educativas; puesto que, este tipo de programas engloban una gran variedad de herramientas tecnológicas, que interactúan a la par con técnicas didácticas como la Gamificación permitiendo tanto al docente como a los alumnos la fácil comprensión de cualquier tema propuesto. GarcíaPeñalvo y Seoane Pardo (2015) señalan que estas herramientas informáticas están 
desarrolladas de manera que ayudan notoriamente a la educación a la distancia en función de objetivos integrales, que buscan mejorar la experiencia virtual de los actores educativos, permitiendo el acceso a información imprescindible que ayuda a una mejor asimilación de conocimientos.

De igual modo, es importante conocer lo que es la Gamificación; puesto que es una técnica que propicia el aprendizaje por medio del juego, con el objetivo de obtener mejores resultados en los alumnos sobre una temática 0 asignatura de difícil comprensión. Así mismo, el investigador Quintanal-Pérez (2016) explica que la Gamificación consiste en aplicar conceptos y dinámicas propias del diseño de juegos que estimulan y hacen más atractiva la interacción del alumno con el proceso de aprendizaje, con el objetivo de provocar ambientes agradables, que genere un compromiso con la actividad en la que participa y que apoye al logro de experiencias positivas para alcanzar un aprendizaje significativo.

Es esta misma línea definimos Educaplay según Albarracín-Villamizar et al. (2020) es una plataforma online gratuita que posibilita la creación de una gran variedad de actividades ligadas a la educación, fomenta el interés del alumnado mediante la creación de actividades multimedia; se centra en dos bloques de actividades, las propuestas por el internauta y las ya existentes en la plataforma. Para su utilización basta con ingresar a su página electrónica, lo que la convierte en una herramienta esencial dentro del campo educativo.

Ventajas de usar Educaplay

- No es necesario descargar un software de instalación.

- Posibilita la interacción de varias plataformas LMS.

- La interacción de la plataforma es de fácil comprensión

- Se puede crear un sinnúmero de actividades y se las puede extraer. Actividades de Educaplay

- Sopa de letras, puzles, presentaciones, adivinanzas, crucigramas, Diálogos, Dictados, Test, Mapas, video Quizz 


\section{Normativa Vigente de la Educación en Ecuador}

La sustentación dentro del marco legal de esta investigación está determinada por la constitución del Ecuador, la cual vela por el cumplimento y la correcta aplicación de los principios constitucionales en el sistema nacional de educación, la cual en el artículo dieciséis numeral dos, sección tercera de la constitución del Constitución de la República del Ecuador (2008) establece:

- Art. 16.- Todas las personas, en forma individual o colectiva, tienen derecho a: El acceso universal a las tecnologías de información y comunicación.

- Art. 25.- Las personas tienen derecho a gozar de los beneficios y aplicaciones del progreso científico y de los saberes ancestrales.

- Art. 26.- La educación es un derecho de las personas a lo largo de su vida y un deber ineludible e inexcusable del Estado. Constituye un área prioritaria de la política pública y de la inversión estatal, garantía de la igualdad e inclusión social y condición indispensable para el buen vivir. Las personas, las familias y la sociedad tienen el derecho y la responsabilidad de participar en el proceso educativo.

Si bien es cierto que el estado tiene la obligación de proveer de recursos tecnológicos (Hardware) a las instituciones educativas del país, pero lo más importante sería capacitar a los actores educativos para que operen con lógica los programas (Software); de esta manera podrán analizar información relevante, que les ayude a desarrollar y utilizar plataformas que generan interés en ellos, fomentando una cultura de innovación para el desarrollo de nuevas tecnologías en el proceso de aprendizaje.

\section{Técnicas empleadas en la investigación}

En primera instancia, en lo que tiene que ver con el Pretest y el Pos test; RodríguezConde et al. (2017) explican que generalmente se utilizan mediante la aplicación de cuestionarios, que permiten conocer el comportamiento o nivel de aprendizaje adquirido sobre un determinado tema a un grupo de individuos. La mecánica de este tipo de 
investigación, posibilita a que su uso pueda darse antes o después de aplicar el tratamiento experimental en el grupo tratante, con el objetivo de saber la situación actual y las metodologías que serán necesarios desarrollar para el caso de estudio.

Así mismo, para recoger información sobre el funcionamiento de la herramienta Educaplay y su aplicabilidad en matemática, es necesario acudir a grupos de docentes conocedores. Según Robles-Garrote y Rojas (2015) las entrevistas a expertos sirven para determinar la autenticidad de la información recopilada sobre un tema específico, además permiten al investigador recopilar información de la cual no tiene un completo conocimiento; puesto que, posibilita interactuar con un grupo de individuos que tienen un real conocimiento sobre la problemática sujeta a investigación; así de esta manera, se lograra una mayor comprensión y aplicación del tema de estudio.

De igual manera, para la recolección de los resultados una vez desarrollada la implementación de la plataforma, es necesario realizar un cuestionario que permitirá conocer y analizar los datos de forma coherente e integral, puesto que este tipo de instrumento posibilita la recolección de información cuantitativa, con el propósito de corroborar las hipótesis planteadas para el desarrollo de esta investigación como válidas o nulas.

\section{METODOLOGÍA}

El presente trabajo tiene un alcance explicativo, en primera instancia se elaboró una revisión minuciosa de material bibliográfico referente al tema de estudio (Erazo, 2021). Posteriormente se realizó un estudio de campo mediante las siguientes técnicas e instrumentos de evaluación; Entrevistas a docentes expertos, Pre Test, Post Test.

La técnica que se utilizó para la recolección de información del paradigma epistemológico antes de aplicar la herramienta Educaplay es un Pre-Test; en este sentido, en la primera parte del documento se investigó de qué manera los docentes están implementando las metodologías y la didáctica en los procesos de enseñanza de la institución; así mismo, en segunda instancia se elaboraron preguntas para conocer el nivel de entendimiento de 
los alumnos sobre plataformas educativas; finalmente, se evaluaron los conocimientos que poseen los educandos sobre algebra.

Posteriormente, mediante Microsoft Forms se realizaron entrevistas a un grupo de tres docentes conocedores de la aplicabilidad de Educaplay en la asignatura de Matemática; con el propósito de profundizar el conocimiento sobre la herramienta digital y el tipo de actividades que dispone para lograr una mejor comprensión de Algebra. En este sentido, una vez desarrollada esta etapa; se continuó el proceso con la elaboración de las actividades disponibles en la plataforma; las mismas que fueron direccionadas a la fácil comprensión de la temática.

Finalmente, se procedió con la implementación de un cuestionario, que ayudó a medir con veracidad que tanto influye la aplicación de la plataforma como estrategia didáctica de aprendizaje de algebra. Antes de aplicar los instrumentos se precedió a realizar las autorizaciones necesarias en la institución; de la misma manera se firmaron cartas de consentimiento para la aplicación de los cuestionarios al tratarse de estudiantes menores de edad, cumpliendo con los parámetros legales necesarios.

Por otra parte, la muestra está comprendida por 22 alumnos pertenecientes al noveno año de básica de la Unidad Educativa Mariscal Antonio José de Sucre de la parroquia Rivera, ubicada en el cantón de Azogues de la provincia del Cañar, periodo lectivo 20212022. Los alumnos se encuentran en un rango de edad de 13 a 14 años. Para el análisis de datos se utilizó la aplicación Surver Monkey, la cual mediante un cálculo minucioso tomo en cuenta el nivel de confiabilidad del $95 \%$ y el margen de error del $5 \%$.

En lo que tiene que ver con los instrumentos aplicados a los alumnos; para el pre test se realizó un total de 12 preguntas estructuradas bajo la escala de Likert, fueron validadas con herramientas digitales y análisis estadístico inferencial mediante la ayuda del programa SPSS. Al realizar la prueba de confiabilidad mediante el coeficiente Alfa de Cronbach se obtuvo un valor de 0.765 por lo que se prosiguió con la aplicación del cuestionario. 


\section{RESULTADOS}

A continuación, se presentan los resultados de la encuesta aplicada a los alumnos luego de la aplicación de la plataforma Educaplay, los mismos responden al objetivo de investigación: analizar de qué manera la Herramienta Educaplay puede aportar en el proceso de aprendizaje de algebra en los estudiantes de Octavo Año de Básica de la Unidad Educativa Mariscal Antonio José de Sucre. Las preguntas se estructuraron en base a tres dimensiones principales como son; Motivación y Metodología, Conocimiento de Herramientas Digitales y Conocimientos de algebra.

Además, es importante recalcar que se realizó un análisis mediante una prueba de normalidad a cada una de las preguntas del cuestionario, al estar comprendida la muestra por 22 datos se utilizó la prueba de Shapiro Wilk, obteniendo en todos los análisis valores menores a 0,05 respectivamente, comprobando que todas las variables son normales, por lo tanto, son paramétricas. por lo tanto, para el análisis en primer lugar se estableció la siguiente regla de decisión.

\section{Regla de decisión}

- Si el nivel de Significancia ( $p$ valor) es mayor que 0,05 se asume Ho

- Si el nivel de significancia ( $p$ valor) es menor que 0,05 se asume $\mathrm{H} 1$

\section{Tabla1.}

Nivel de motivación y aprendizaje en el aula con recursos tradicionales e innovadores.

\begin{tabular}{clcccc}
\hline & Pres test - Pos test & N & Media & $\begin{array}{c}\text { Desviación } \\
\text { típ. }\end{array}$ & Sig. (bilateral) \\
\hline $\begin{array}{c}\text { Frecuencia motivación } \\
\text { docente }\end{array}$ & $\begin{array}{l}\text { Recursos tradicionales } \\
\text { Recursos Innovadores }\end{array}$ & 22 & 3,86 & 1,390 & 0,002 \\
\hline $\begin{array}{c}\text { Frecuencia con la que } \\
\text { los docentes logran } \\
\begin{array}{c}\text { aprendizaje } \\
\text { significativo }\end{array}\end{array}$ & Recursos tradicionales & 22 & 4,66 & 0,351 & 0,003 \\
\hline
\end{tabular}

Fuente: Cuestionario. 
En la tabla 1 se encuentra la frecuencia del nivel de motivación con recursos tradicionales en contraparte con los recursos Innovadores, en donde se observa que existe un incremento en la media; puesto que, con recursos tradicionales es de 3,86 mientras que con recursos innovadores es de 4,66 respectivamente.

Así mismo se aprecia que el nivel significancia bilateral es de 0,002 y 0,003 en cada uno de los dos momentos, por lo tanto, se cumple que $p$ es menor que 0,05 y se asume la hipótesis alterna $\mathrm{H} 1$; Indicando que existe un incremento en la motivación cuando se utiliza Educaplay.

Así mimo en la tabla 1 se muestra el nivel de frecuencia con la que los docentes logran generar conocimientos duraderos con recursos tradicionales en contraparte con los recursos Innovadores, en donde se observa que existe un aumento en la media; puesto que, con recursos tradicionales es de 4,05 mientras que con recursos innovadores es de 4,91 respectivamente.

Así mismo se aprecia que el nivel significancia bilateral es de 0,003 y 0,004 en cada uno de los dos momentos, por lo tanto, se cumple que $p$ es menor que 0,05 y se asume la hipótesis alterna $\mathrm{H} 1$; por lo tanto, la utilización de la herramienta genera mayor asimilación de conocimientos en los educandos.

\section{Tabla 2.}

Conocimiento de plataformas educativas y Educaplay

\begin{tabular}{cccccc}
\hline & Test global & $N$ & Media & $\begin{array}{c}\text { Desviación } \\
\text { típ. }\end{array}$ & Sig. (bilateral) \\
\hline $\begin{array}{c}\text { Conocimiento de } \\
\text { herramientas } \\
\text { educativas }\end{array}$ & Pretest & 22 & 2,18 & 1,402 & 0,000 \\
\hline $\begin{array}{c}\text { Conocimiento de } \\
\text { Educaplay }\end{array}$ & Postest & 22 & 4,45 & 0,739 & 0,000 \\
\hline
\end{tabular}

Fuente: Cuestionario 
En la tabla 2 se muestra el nivel de conocimiento de los alumnos sobre plataformas educativas en los dos momentos de la investigación, en donde se observa mediante el test global que existe un incremento en la media; puesto que, en el pre test es de 2,18 mientras que posteriormente con el Post test es de 4,45 respectivamente. Por lo tanto, se observa claramente que los alumnos no utilizaron en gran medida plataformas educativas en las aulas de clase.

De igual modo, se aprecia que el nivel significancia bilateral es de 0,000 y 0,000 en cada uno de los dos momentos, por lo tanto, se cumple que $p$ es menor que 0,05 y se asume la hipótesis alterna $\mathrm{H} 1$; En consecuencia, la utilización de la herramienta genera mayor interés por conocer y realizar actividades en plataformas digitales, antes que de forma tradicional.

También En la tabla 2 se muestra el nivel de conocimiento de Educaplay por parte de los alumnos en el área de matemática en los dos momentos de la investigación, en donde se observa que existe un incremento en la media; puesto que, en el pre test es 2,05 mientras que con el post test es de 4,18 respectivamente. Por lo tanto, se observa claramente que los docentes no utilizaron la herramienta en los procesos de enseñanza y los alumnos no conocían Educaplay ni utilizaron esta herramienta en las aulas de clase. Así mismo se aprecia que el nivel significancia bilateral es de 0,000 y 0,000 en cada uno de los dos momentos, por lo tanto, se cumple que $p$ es menor que 0,05 y se asume la hipótesis alterna $\mathrm{H} 1$; por lo tanto, la utilización de la herramienta provoca un mayor interés por aprender y realizar actividades por medio de esta metodología. 


\section{Tabla 3.}

Nivel de conocimiento de algebra recursos tradicionales e innovadores.

\begin{tabular}{cllccc}
\hline & \multicolumn{1}{c}{ Test global } & N & Media & $\begin{array}{c}\text { Desviación } \\
\text { típ. }\end{array}$ & Sig. (bilateral) \\
\hline $\begin{array}{c}\text { Conocimiento de } \\
\begin{array}{c}\text { Expresiones } \\
\text { algebraicas }\end{array}\end{array}$ & Recursos tradicionales & 22 & 3,18 & 1,259 & 0,000 \\
\hline $\begin{array}{c}\text { Conocimiento de } \\
\text { valores Numéricos }\end{array}$ & Recursos tradicionales & 22 & 3,45 & 0,912 & 0,001 \\
\hline $\begin{array}{c}\text { Conocimiento de } \\
\text { identidades notables }\end{array}$ & Recursos innovadores & 22 & 4,59 & 0,503 & 0,000 \\
\hline $\begin{array}{c}\text { Conocimiento de } \\
\text { Ecuaciones }\end{array}$ & Recursos innovicionales & 22 & 3,09 & 1,109 & 0,000 \\
algebraicas & Recursos innovadicionales & 22 & 3,45 & 1,057 & 0,002 \\
\hline
\end{tabular}

Fuente: Cuestionario.

En la tabla 3 se muestra el nivel de conocimiento de algebra de los alumnos en los dos momentos de la investigación, en lo que tienen que ver con expresiones algebraicas existe un incremento de la media de 3,18 a 4,36 respectivamente. Por ende, se aprecia que el nivel significancia bilateral es de 0,000 y 0,001 en cada uno de los dos momentos, por lo tanto, se cumple que $\mathrm{p}$ es menor que 0,05 y se asume la hipótesis alterna $\mathrm{H} 1$; por lo tanto, la correcta utilización e implementación de la plataforma Educaplay si ayuda a incrementar la comprensión de esta temática.

En lo que tienen que ver con valores numéricos existe un incremento de la media de 3,45 a 4,59 respectivamente. En consecuencia, se aprecia que el nivel significancia bilateral es de 0,000 y 0,000 en cada uno de los dos momentos, por lo tanto, se cumple que p es menor que 0,05 y se asume la hipótesis alterna $\mathrm{H} 1$; por lo tanto, la correcta utilización e implementación de la plataforma Educaplay si ayuda a incrementar la comprensión de la temática. 
En lo que tienen que ver con identidades notables existe un incremento de la media de 3,09 a 4,68 respectivamente. Por ende, se aprecia que el nivel significancia bilateral es de 0,000 y 0,002 en cada uno de los dos momentos, por lo tanto, se cumple que $p$ es menor que 0,05 y se asume la hipótesis alterna $\mathrm{H} 1$; por lo tanto, la correcta utilización e implementación de la plataforma Educaplay si ayuda a incrementar la comprensión de la temática.

En lo que tienen que ver con ecuaciones algebraicas existe un incremento de la media de 3,45 a 4,45 respectivamente. Por ende, se aprecia que el nivel significancia bilateral es de 0,000 y 0,000 en cada uno de los dos momentos, por lo tanto, se cumple que $p$ es menor que 0,05 y se asume la hipótesis alterna $\mathrm{H} 1$; por lo tanto, la correcta utilización e implementación de la plataforma Educaplay si ayuda a incrementar la comprensión de la temática.

\section{PROPUESTA}

El enfoque de la investigación estuvo orientado a determinar si la incorporación de la plataforma educativa Educaplay en los procesos de aprendizaje de algebra mejora la comprensión y asimilación de conocimientos; una vez determinada la hipótesis como válida al obtener resultados positivos, se realiza una guía metodológica para la utilización de la plataforma Educaplay en cada uno de los subniveles educativos de la Institución Educativa "Mariscal Antonio José de Sucre".

\section{Actores presentes en la elaboración de la guía:}

- Responsables: Rectora y Docentes de la institución

- Beneficiarios: Alumnos y Docentes de la Institución

- Financiamiento: Ninguno

- Recursos: proyector, internet, plataforma Educaplay, computadoras

- Periodo de Aplicación: Lectivo 2021-2022 


\section{Revista Interdisciplinaria de Humanidades, Educación, Ciencia y Tecnología}

Año VII. Vol. VII. N³. Edición Especial III. 2021

Hecho el depósito de ley: pp201602FA4721

ISSN-L: 2542-3029; ISSN: 2610-802X

Universidad Nacional Experimental Francisco de Miranda (UNEFM). Santa Ana de Coro. Venezuela

Cristian Fernando Álvarez-Guamán; Juan Carlos Erazo-Álvarez

\section{Objetivos}

- Dinamizar los procesos de enseñanza- aprendizaje de algebra en la institución.

- Incorporar el uso de tecnologías en los docentes y alumnos.

- Desarrollo de Metodologías que llaman la atención de los estudiantes.

Es importante que tanto los docentes como los alumnos obtengan conocimientos solidos sobre la utilización de recursos tecnológicos dinamizadores como plataformas educativas, por lo tanto, con la guía denominada ECPEE (Elaboración, Capacitación, Planificación, Ejecución, Evaluación); se busca que los actores educativos desarrollen los procesos de aprendizaje de manera innovadora, con el propósito de llegar a cambios positivos para la educación actual.

\section{Tabla 4.}

Etapas de desarrollo de la guía.

\begin{tabular}{|c|c|c|c|}
\hline Etapas & Objetivo & Actividad & Responsable \\
\hline Elaboración & $\begin{array}{l}\text { Desarrollo de una guía } \\
\text { que oriente como } \\
\text { desarrollar actividades } \\
\text { en la plataforma } \\
\text { educativa. }\end{array}$ & $\begin{array}{l}\text { Describir cada una de } \\
\text { las actividades } \\
\text { disponibles en la } \\
\text { plataforma Educaplay y } \\
\text { como pueden ser } \\
\text { ejecutados en el aula. }\end{array}$ & Rector -Docente \\
\hline Inducción & $\begin{array}{c}\text { Socializar con los } \\
\text { docentes de la } \\
\text { institución las } \\
\text { bondades de la } \\
\text { herramienta y su } \\
\text { factibilidad para el } \\
\text { proceso de enseñanza. }\end{array}$ & $\begin{array}{l}\text { Realizar una variedad } \\
\text { de actividades } \\
\text { disponibles en la } \\
\text { plataforma Educaplay }\end{array}$ & Docente \\
\hline Planificación & $\begin{array}{l}\text { Implementación de las } \\
\text { actividades de } \\
\text { plataforma en el PUD. }\end{array}$ & $\begin{array}{l}\text { Desarrollar actividades } \\
\text { mediante la plataforma } \\
\text { en los momentos de la } \\
\text { clase } \\
\end{array}$ & Docente \\
\hline Ejecución & $\begin{array}{c}\text { Desarrollar actividades } \\
\text { lúdicas como sopa de } \\
\text { letras, Puzzles, } \\
\text { crucigramas, videos, } \\
\text { juegos. }\end{array}$ & $\begin{array}{l}\text { Los alumnos } \\
\text { Desarrollan actividades } \\
\text { disponibles en } \\
\text { Educaplay. }\end{array}$ & Docente -Alumno \\
\hline
\end{tabular}


Elaboración: Los autores.

\section{CONCLUSIONES}

Por medio de la investigación se llega la conclusión que la incorporación de Educaplay en el proceso de aprendizaje de algebra, ayuda en gran medida a la mejor comprensión y asimilación de conocimientos; además genera en los alumnos un mayor interés en el aula de clase, esto debido a la didáctica de las actividades disponibles en la herramienta, la misma que llama su atención; sin embargo, es necesario que tanto los docentes como los alumnos conozcan el correcto funcionamiento de la plataforma, para que las actividades pedagógicas son direccionadas de forma correcta y no generen complicaciones o inconvenientes.

En los resultados obtenidos se puede visualizar el poco entendimiento de los docentes sobre redes digitales; este desconocimiento es aún mucho mayor en los estudiantes; puesto que, ni siquiera han utilizado este tipo de plataformas en ninguna asignatura perteneciente a nuestro currículo nacional; por lo tanto, es necesario que el ministerio de educación tenga en cuenta estos tipos de software con el fin de que los alumnos tengan nuevas alternativas en el proceso de aprendizaje.

Asimismo, a través del análisis de las diferentes investigaciones sobre la temática abordada en este trabajo, se buscó medir el nivel de conocimiento por parte de la comunidad educativa sobre plataformas educativas digitales, con el propósito de que su implementación sea considerada en las aulas de clase de las instituciones educativas, mediante propuestas que salgan de lo monótono y tradicional; En este sentido, los aprendizajes en las diferentes áreas presentes en la educación serán más enriquecedores y generaran un mayor interés en el proceso de enseñanza-aprendizaje. 
Por todo la antes expuesto se elabora una propuesta diseñada en forma secuencial mediante una guía modelo que consta de las siguientes partes: Elaboración, Capacitación, Planificación, Ejecución, Evaluación (ECPEE), en donde se platea que los docentes realicen cada uno de estos pasos de forma ordenada, de esta manera el proceso de enseñanza será más efectivo y generara una mayor asimilación de conocimientos en los alumnos permitiendo que el aprendizaje sea dinámico y proactivo.

\section{REFERENCIAS CONSULTADAS}

Albarracín-Villamizar, C. Z., Hernández- Suárez, C. A., \& Rojas -Suárez, J. P. (2020). Objeto Virtual De Aprendizaje Para Desarrollar Las Habilidades Numéricas: Una Experiencia Con Estudiantes De Educación Básica [Virtual Learning Object To Develop Numeracy Skills: An Experience With Elementary School Students]. Panorama, 14(26), 111-133. https://doi.org/10.15765/pnrm.v14i26.1486

Badia-Garganté, A., Meneses-Naranjo, J., \& García-Tamarit, C. (2015). Technology use for teaching and learning [Uso de la tecnología para la enseñanza y el aprendizaje]. Píxel-Bit. Revista de Medios y Educación, O(46). https://n9.cl/deayo

Baldor, A. (2010). Álgebra de Baldor [Baldor's algebra ]. https://n9.cl/wtnm

Boude-Figueredo, Ó., \& Sarmiento, J. (2016). Herramientas web 2 . 0 : efecto en los aprendizajes de los jóvenes colombianos [Tools Web 2 . 0 : Impact on Learning of Colombian Youths]. Opción, 11, 143-163. https://n9.cl/41ez

Cencia-Crispín, O., Carreño-Colchado, M. Mi., Elche-Querevalú, P., Barrantes-Morales, G. I., \& Cardenas-Baldeon, G. G. (2021). Estrategias docentes de profesores universitarios en tiempos de Covid - 19 [Teaching strategies of university professors in times of Covid-19 ]. Horizonte de La Ciencia, 11(20), 347-360. https://n9.cl/c78ry

Collaguazo-Alvarez, M. E., \& Maggi-Barba, M. A. (2020). Aplicación de la Técnica Informática Educaplay como Estrategia para el Aprendizaje de las Biomoléculas, en los Estudiantes de Bachillerato de la Unidad Educativa Andrés F. Córdova - Cañar, Ecuador [Application of the Educaplay Computer Technique as a Stra. Revista Scientific, 2, 12-26. https://n9.cl/nv5aq

Constitución de la Republica del Ecuador. (2008). [Constitution of the Republic of Ecuador 2008]. Incluye Reformas, 1-136. https://n9.cl/hd0q 
Ducuara-Amado, L. Y., Rodríguez- HernándezRodriguez, A. A., Niño-Vega, J. A., \& Fernández- Morales, F. H. (2020). Material educativo gamificado para la enseñanzaaprendizaje de conceptos de ecología en estudiantes de educación media [Gamified educational material for the teaching-learning of ecology concepts in middle school students]. Revista Boletín Redipe, 9(6), 144-156. https://n9.cl/qoeq2

Erazo Álvarez, J. C. (2021). Capital intelectual y gestión de innovación: Pequeñas y medianas empresas de cuero y calzado en Tungurahua-Ecuador. Revista De Ciencias Sociales, 27, 230-245. Recuperado a partir de https://produccioncientificaluz.org/index.php/rcs/article/view/37004

Faria, R. W. S. de C., \& Maltempi, M. V. (2019). Mathematical Intradisciplinarity with GeoGebra in school mathematics [Intradisciplinariedad matemática con GeoGebra en matemáticas escolar]. Bolema. https://n9.cl/g5d8m

García-Peñalvo, F. J., \& Seoane Pardo, A. M. (2015). An updated review of the eLearning concept. Ten Aniversary[Una revisión actualizada del concepto de eLearning. Decimo aniversario]. Education in the Knowledge Society (EKS), 16(1), 119-144. https://doi.org/10.14201/eks2015161119144

Holguín-García, F. Y., Holguín-Rangel, E. G., \& García-Mera, N. A. (2020). Gamificación en la enseñanza de las matemáticas: Una revisión sistemática. [Gamification in mathematics education: a systematic review]. ELOS: Revista de Estudios Interdisciplinarios En Ciencias Sociales, 22(1), 62-75. https://n9.cl/namlg

Labrador Ruiz de la Hermosa, E., \& Villegas- Portero, E. (2016). Unir Gamificación y Experiencia de Usuario para mejorar la experiencia docente [Uniting Gamification and User Experience to enhance the teaching experience]. RIED. Revista Iberoamericana de Educación a Distancia, 19(2). https://n9.cl/5kwf

Lam-Díaz, R. M. (2016). La redacción de un artículo científico [ Writing a scientific paper]. Revista Cubana de Hematologia, Inmunologia y Hemoterapia, 32(1), 57-69. https://n9.cl/6hsof

Organización de las Naciones Unidas Para la Educación la Ciencia y la Cultura [UNESCO]. (2015). Los profesores de las zonas rurales de Myanmar utilizan las TIC móviles[Teachers in rural Myanmar use mobile Tic ]. https://n9.cl/kja7s 
Organización para la Cooperación y el Desarrollo Económico [OCDE ]. (2021). Respuestas educativas a COVID-19: Adoptar el aprendizaje digital y la colaboración en línea[ Educational responses to COVID-19: Embracing digital learning and online collaboration]. https://n9.cl/54i0k

Salinas-Gañango, J. \& Salvati, A. (2020). Educaplay as an interactive teaching resource aimed at students of the Marketing subject. https://doi.org/10.33996/franztamayo.v2i4.297

Ortiz-Colón, A.-M., Jordán, J., \& Agredal, M. (2018). Gamificación en educación: una panorámica sobre el estado de la cuestión. [Gamification in education: an overview of the state of the art]. Educação e Pesquisa, 44(0), 1-17. https://n9.cl/wg2ug

Programa Internacional para la Evaluación de Instituciones[ PISA-D]. (2018). Informe general PISA 2018 - Banco de Información [PISA 2018 General Report - Information Bank]. https://n9.cl/xwy5b

Quintanal-Pérez, F. (2016). Aplicación de herramientas de gamificación en física y química de secundaria [Applying of gamification tools in physics and chemistry of secondary education]. Serbiluz, 32, 327-348. https://n9.cl/1f1g3

Robles- Garrote, P., \& Rojas, M. del C. (2015). La validación por juicio de expertos: dos investigaciones cualitativas en Lingüística aplicada [Validation by expert judgements: two cases of qualitative research in Applied Linguistics ]. Nebrija, 1-16. https://n9.cl/u0vb

Rodríguez-Conde, M. J., García-Peñalvo, F. J., \& García-Holgado, A. (2017). Pretest y postest para evaluar la implementación de una metodología activa en la docencia de Ingeniería del Software [Pretest and posttest to evaluate the implementation of an active methodology in the teaching of Software Engineering]. Zenodo, 1-17. https://doi.org/10.5281/ZENODO.1034822

Vaillant, D., Zidán-Rodríguez, E., \& Biagas-Bentacor, G. (2020). Uso de plataformas y herramientas digitales para la enseñanza de la Matemática [Use of digital platforms and tools for mathematics teaching]. Ensaio: Avaliação e Políticas Públicas Em Educação, 28(108), 1-23. https://n9.cl/pxbil

Vargas-Murillo, G. (2017). Recursos educativos didácticos en el proceso enseñanza aprendizaje[Didactic educational resources in the teaching-learning process ]. https://n9.cl/jed4i 
Revista Interdisciplinaria de Humanidades, Educación, Ciencia y Tecnología

Año VII. Vol. VII. N³. Edición Especial III. 2021

Hecho el depósito de ley: pp201602FA4721

ISSN-L: 2542-3029; ISSN: 2610-802X

Universidad Nacional Experimental Francisco de Miranda (UNEFM). Santa Ana de Coro. Venezuela

Cristian Fernando Álvarez-Guamán; Juan Carlos Erazo-Álvarez

(O2021 por los autores. Este artículo es de acceso abierto y distribuido según los términos y condiciones de la licencia Creative Commons Atribución-NoComercial-Compartirlgual 4.0 Internacional (CC BY-NC-SA 4.0)

(https://creativecommons.org/licenses/by-nc-sa/4.0/). 